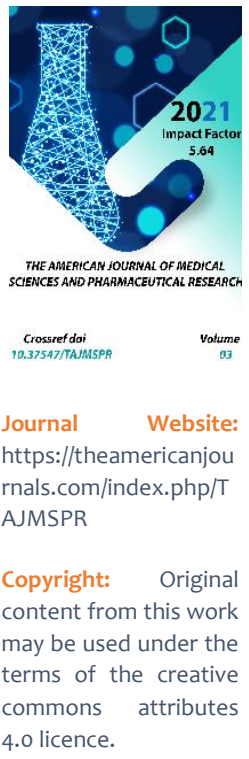

\title{
Morphofunctional Changes Of The Thymus Under The Influence Of Various Environmental Factors
}

\author{
N.H.Asadova \\ Bukhara State Medical Institute, Republic of Uzbekistan, Bukhara
}

\section{ABSTRACT}

The study of the literature has shown that the morphological status of the thymus has been studied superficially in many works. The cellular composition of the thymus parts under the influence of physical or chemical environmental factors in the age aspect has not been studied enough. All this requires a more detailed study of the morphological changes occurring in this organ under the influence of various factors.

\section{KEYWORDS}

Thymus, Environment, Morphology, External Factors.

\section{INTRODUCTION}

Subsequently, in the first decade of the XXI century, experimental studies were conducted that demonstrated the participation of the thymus in balancing the activity of the hypothalamic-pituitary-adrenal axis by limiting the stress-damaging effect when it is excessively activated [8]. Moreover, at the turn of the $X X$ and $X X I$ centuries, thanks to the success of histochemistry, it was discovered that the same signaling molecules are found in the cells of the endocrine, nervous and immune systems, and these systems "talk" to each other in the same language - the language of signaling molecules [37]. All these data point to the great importance of the thymus for living organisms. However, the in vivo study of this organ in humans is a difficult task, since, on the one hand, it can quickly change its size, on the other-there are no available methods for studying its functional activity. The latter was the reason for conducting the present study. 
The thymus in humans, starting to function from the 4th week of embryogenesis, reaches a significant size by the time of birth (allowing it to be visualized using ultrasound[2].

\section{Functional morphology of the thymus.}

Subcapsular zone, which occupies area of $1 / 4$ of the crust beneath the basal membrane of the lobules presented a continuous layer of epithelial cells lying on the basal membrane under the capsule and around blood vessels cortical areas. Early thymocytes, fibroblasts, and a few macrophages are located in the cells of the epithelial network of this zone. It is assumed that the purpose of the subcapsular zone is to create a microenvironment for $\mathrm{T}$ lymphocyte precursors that migrate to the thymus from the bone marrow for their proliferation and the very initial stages of maturation.

The inner cortical zone consists of a broadleaved network of epithelial cells, in the "reservoirs" of which further maturation of Tlymphocytes occurs. Epithelial cells of this zone express class I and II antigens of the HLA system, here the thymocyte $\mathrm{T}$-cell receptor is formed, and mature T-lymphocytes of this zone carry homing receptors for migration to T-dependent zones of peripheral lymphoid organs. The population of lymphocytes in this zone is represented by $60-80 \%$ of all thymus lymphocytes, most of which are not mature, but there are also mature T-lymphocytes. It is assumed that the functional purpose of this zone is to further differentiate the $T$ lymphocytes entering it.

\section{THE MAIN FINDINGS AND RESULTS}

The medullary zone is the medullary substance of the lobules. This zone is represented by a dense network of large epithelial cells, the processes of which are connected to each other by desmoconnected contacts. In the medullary zone there is a large number of interdigitating (dendritic) cells, and closer to the cortical-medullary border, macrophages rich in lysosomes and phagolysosomes accumulate. In the same zone are myoid cells that have acetylcholine receptors, as well as "sphinx cells" with signs of both epithelial and myoid cells. The thymic bodies (Gassal's bodies) are located in the same zone. Morphologically mature T-lymphocytes are brought here for further antigen-dependent maturation. The lymphocytes of the brain zone have a mature phenotype and are functionally active. These are immunocompetent cells that enter the bloodstream. The function of the medullary zone is to provide antigendependent maturation of T-lymphocytes due to the action of thymic hormones of epithelial cells, direct contact with interdigitating cells, and the influence of interleukins [32].

The intra-lobular perivascular spaces (VPP) are the zones surrounding all the intra-lobular vessels of the thymus. The boundaries of the zone are, on the one hand, the basal membrane of the vessels, on the other-the basal membrane of the epithelial cells of the parenchyma of the thymus. In function intralobular perivascular spaces include transport of t-lymphocytes and the establishment of the structural basis gematologicheskogo barrier. In general, the cellular composition of the VPP is close to the peripheral lymphoid tissue, and changes in them during the development of pathology are similar to those in the lymph nodes [28].

One of the most reactive, fast-reacting systems of the body to the effects of damaging factors at the earliest stages is the human immune system. It is formed by a complex of organs and tissues that provide protection from 
foreign endo - and exogenous influences $[6,12$, $23,26,30]$.

More than 100 years ago, it was found that the thymus as a large organ is normally detected only in young, but not in sexually mature individuals. The same has been identified and the person in the conduct of postmortem and forensic studies. After the end of puberty, the thymus in adolescents begins to undergo agerelated involution. Nevertheless, one important feature of the thymus in adults should be noted: despite the ongoing involutional processes, the function of this organ continues to persist until death [15]. The immune system originated in the early stages of evolution and its activity is based on the recognition of foreign antigens, their destruction and removal, which is extremely necessary for the survival of the organism [15].

The main site for the development of specific immunological reactions is the lymphoid tissue, which contains numerous cell populations involved in ensuring the genetic constancy of the internal environment of the body [17].

The thymus as the primary organ of the immune system largely determines not only the state of the peripheral organs of immunogenesis, but also the severity of the protective reactions of the entire body [14]. The regulatory and censor role of the thymus in immunogenesis is related to the state of its reticuloepithelium and lymphocytes. Thymic cells (Gassal cells) produce the humoral factor of the thymus, which determines the immune competence of the lymphoid tissue [31].

The central immune organ, the state of which largely depends on the severity of the protective reactions of the entire body, is the thymus. It was revealed that the bioaminecontaining structures of the thymus regulate the processes of immunogenesis directly. The thymus is the central organ of mammalian immunogenesis, where T-lymphocytes form and multiply, and the red bone marrow, where B-lymphocytes form and multiply. The lymph nodes, spleen, tonsils, and lymphoid follicles of the intestine are peripheral lymphoid organs $[22,24]$.

The thymus is considered as an immune organ in which natural and acquired immunity is formed with the help of biologically active peptides [6].

The thymus in the structure of the immune system ensures the maturation and differentiation of T-lymphocytes, including in peripheral immune organs, and stimulates the integration of various populations of $\mathrm{T}$ lymphocytes and macrophages for the implementation of immune responses [27,39].

Studies of the thymus of people of different ages, which were performed during autopsy, revealed the expression of serotonin in human thymus cells at all stages of ontogenesis. An increase in the number of cells containing serotonin in elderly people and the preservation of this hormone in senile people and long-livers at the same level as in the early stages of ontogenesis were found. The intensity of serotonin synthesis during ontogenesis does not change. The data obtained indicate the preservation of the endocrine function of the gland during aging [24].

In the thymus, as in the body as a whole, all cells are in close contact with each other interaction with each other. Intercellular information interactions are the main integrative and coordinating systems of the body. The communication connection of the cell is carried out with the help of signaling 
molecules. These include ions, gases, peptides, peptide hormones, metabolites, and steroids. The receptors that are located either on the plasma membrane or inside the cell are the receivers and transmitters of the signaling molecules. The thymus is one of those organs in which molecular and cellular neuroimmunoendocrine interactions are most clearly manifested, which play a key role in providing both local and general biological effects $[3,13]$.

It is known that the development of cancer depends on genetic causes, as well as on hormonal regulation, immune responses of the body and other factors. However, recently, scientists have been paying the most attention to the study of the state of immunity. It was revealed that the central organ of the immune system, the thymus, not only with age, but also with a number of diseases, including various infections, severe injuries, and malignant neoplasms, undergoes atrophic changes [11]. It has been established that the tumor process in the body leads to the development of acquired immunodeficiency [26].

The immune system is a unique defense mechanism that provides homeostasis, and in contact with any antigen, it not only reacts in the form of a specific immune response, but is also able to involve the nervous and endocrine systems in this process through humoral factors[18].The leading role in such interactions belongs to the thymus, which contains numerous cell types that support the processes of immunogenesis. Morphological changes in the thymus that occur in response to stress, to various antigens, are adaptive and are accompanied by changes in the cytoarchitectonics and microenvironment of cells, which, apparently, is the cause of the development of immunodeficiency in these conditions[35]. Many scientists have studied changes in the thymus after chemotherapy courses $[5,40,35,37]$.

The opinion that the thymus is completely atrophied in adults is erroneous. The complete termination of the function of this organ for the macroorganism means its death. In such cases, death occurs from infectious, oncological or autoimmune diseases or from the inability to resist stress.

Recurrent thymic hyperplasia,an increase in the organ under study after chemotherapy compared to the baseline level, was detected in 20 patients aged $18-53$ years (an average of 33 years). These findings suggest that the adult thymus retains the ability to regenerate after chemotherapy, especially in young adults.Such hyperplasia may contribute to the renewal of thymopoiesis and replenishment of the peripheral T-cell pool after chemotherapy in adults [40].

A study conducted using imunofan showed a decrease in the degree of destruction of lymphocytes, since it has the ability to protect their DNA from damage caused by cyclophosphane[27], thereby reducing the degree of involutional changes in the thymus, and also accelerates the restoration of the thymus structure after acute involution induced by cyclophosphane [5].

It was found that after 3 -fold administration of polyoxidonium in therapeutic doses to mice in the first 14 days, there is an increase in the area of the cortical substance of the thymus with a simultaneous decrease in the area of the medulla [40]. The use of polyoxidonium for 3 weeks reduces the degree of development of accidental involution of the thymus, after removal of the spleen, and contributes to the correction of the immunodeficiency state [19]. 
Under chronic stress, there is a decrease in the number of T-lymphocyte precursors in the red bone marrow and a decrease in the level of their chemoattractants in the thymus, which contributed to organ hypoplasia [36].

In the work of V. H. Havinson (2010), a study of the immune system organs of rats exposed to gamma radiation was carried out. In the irradiated animals, the internal organs were moderately anemic, and the mesenteric lymph nodes were dark in color. The thymus and spleen were reduced in size [20].

The use of ethanol-containing beverages by women, especially during pregnancy, was studied, which was a factor that most unfavorably integrates the causes that led to immunodeficiency conditions, in particular, the thymus in newborns. Studies show that immunodeficiency can be attributed to the persistent manifestations of fetal alcohol syndrome, which often occurs in children due to prenatal exposure to ethanol $[41,37]$.

The growth of a malignant tumor may depend on both cellular and humoral factors of the thymus [11].

There is a hypothesis that during tumor growth, the output of immature thymocytes may increase, which migrates to the tumor, where they support the growth of transformed cells - the so-called lymph dependent growth phase. According to the literature, it is known that the tumor-stimulating effect of immature T-lymphocytes can consist both in increasing the growth of the tumor cells themselves and blood vessels. The growth of neoplasms depends on the thymus not only as an organ that produces lymphocytes, but also as a gland that synthesizes hormones [1].

It is known that the organs of the immune system and, first of all, its central organ, the thymus, responsible for the formation of immunological reactions, play a crucial role in maintaining the homeostasis of the body, in ensuring the stability of its antigenic structures, in constant supervision of the genetic constancy of the body's somatic cells [9].

A little-studied function of the thymus is its participation in the realization of stress. Under stress, the thymus and lymph nodes are reduced. If the stressor is not strong enough, and the body's resistance is high, then these changes may not be detected. Hormones and polypeptides of the thymus are functional antagonists of the stress system. Under the influence of thymus polypeptides, the body's resistance to various stressful influences increases, and the thymus itself is not only the central organ of the immune system, but also the organ of the stress-limiting system [7]. The thymus response to stress, including infectious stress, includes its involution, which is pronounced in children, especially in infants and young children. When conducting ultrasound diagnostics of the thymus in 60 children of the first year of life in the dynamics of the acute infectious process, it was revealed that the thymus value decreases on the 2 nd 3rd day of the disease (ARI, acute pyelonephritis, acute intestinal infections), on the 10th - 14th day from the onset of the disease and after 1.5-2 months [15].

Abscesses and other inflammatory changes in the thymus are extremely rare, since the thymus is sufficiently reliably isolated from participation in daily activities and is involved in inflammation only when it passes to the systemic level or when the damaging agents directly affect the organ itself [16]. 
Atrophy of the thymus parenchyma located around small vessels at the age of 25-40 years reaches $5 \%$ per year $[40,36]$.

The regulatory and censor role of the thymus in immunogenesis is related to the state of its reticuloepithelium and lymphocytes. Thymic cells (Gassal cells) produce the humoral factor of the thymus, which determines the immune competence of the lymphoid tissue [31].

\section{CONCLUSION}

The central organ of the human and animal immune system, the thymus (thymus gland), is an evolutionary acquisition of vertebrates. Its appearance in representatives of this group of living organisms was a key event in the evolution of the immune system [29]. The analysis of the literature shows that in many works the morphological status of the thymus is superficially studied. There is no clear understanding of the study of the cellular composition of parts of the thymus under the influence of physical or chemical environmental factors in the age aspect.

All this requires a deeper study of the morphological changes that occur in this organ under the influence of various factors.

\section{REFERENCES}

1. Babaeva, A. G. Immunology of adaptive growth, proliferation and their disorders. The science.2001-207 p.

2. Budaeva E. K. Echographic characteristics of changes in the thymus gland in normal and pathological conditions in newborns: dis.Candidate of Medical Sciences: 14.00.09/ E. K. Budaeva.M. 2007

3. Fingers $M$. A. Intercellular interactions / $M$. A. Fingers, A. A. Ivanov. - M.: Meditsina, 2003. - 289s.
4. Karaulov A.V. Clinical and immunological efficacy of imunofan in opportunistic infections.The attending physician. 2000. No. 5-6. pp. 28-29.

5. Kiryanov N. A. [et al.] Morphological characteristics of the immune and endocrine systems in endotoxicosis. Medical Bulletin of Bashkortostan. 2013. No. 6. pp. 156-158.

6. Kiseleva N. M. Thymus peptides and their role in the regulation of stress. - Autoref. diss. doctor. biol. nauk. N. M. Kiseleva 2013 - - 46s.

7. Kiseleva, E. P. Mechanisms of thymus involution and activation of the mononuclear phagocyte system in the growth of experimental tumors: Medicine of the Russian Academy of Medical Sciences. -2002. 38 p.

8. Kiseleva N. M. Thymus peptides and their role in stress regulation Sciences. Moscow: 2013. $-46 \mathrm{~s}$.

9. Konstantinova I. V., Babaeva A. G. Twofaced Janus N. M.: "Narconet", 2001.136 p.

10. Kuzmenko L. G. Functional state of the thymus in full-term newborns and their mothers.Journal named after G. $N$. Speransky. - 2011.Vol. 90, No. 1.p. 8-13.

11. Mikhailenko. A. A [et al.]. Preventive Immunology Moscow-Tver, LLC Publishing House "Triada". 2004. 448 p.

12. Nikityuk D. B. "The immune system, stress and immunodeficiency" Moscow: APP Jangar 2000-184 p.

13. Paltsev $M$. A. Neuroimmunoendocrinology of the thymus: manual of Neuroimmunoendocrinology: Meditsina, 2008. - p. 299 - - 345.

14. Sapin M. R. Immune structures of the digestive system Medicine, 2000. 224 p. 
15. Sepiashvili R. I. Functional system of immune homeostasis. Allergology and immunology. 2003. No. 2. 5-14-P

16. Smyslov Z.In New markers of functional activity of the thymus in children dis. cand. med. sci. 14.01.08 2015-45 p.

17. Struchko G. Yu. Polyoxidonium as a corrector of postsplenectomy immunodeficiency state Scientific tr. of the II Congress of physiologists of the CIS. Chisinau: Zdorovye, 2008. P. 159.

18. Trufakin V. A. 2002; L. I. Drozdova et al., 2007; S. N. Lugovskaya et al., 2009).

19. Uddin M. D. The state of the thymus, adrenal glands and thyroid gland in the dynamics of the infectious process in children of the first year of life (according to ultrasound data) abstract of the PhD thesis: 14.00.09 Mohammad Jashim Uddin-2005. - $22 \mathrm{p}$.

20. Khavinson V. H. Prospects for the use of peptide bioregulators to increase the life resource of the body.Journal "Russian Family Doctor" 2010.11 p.

21. Khaitov R. M., Ignatieva G. A. Sidorovich I. G. Medicine Immunology. 2000. 430 p.

22. Khojayan A. B. [et al.] On the immunotoxicity of heavy metal salts. Natural Science and Humanism. 2007. No. 3. pp. 104-105.

23. Khodjayan A. B. Fedorenko. N. N. M. Gevandova.G. Phylogenesis of main organ systems of vertebrates: a textbook for students of I course Stga. Stavropol: Publishing house Stga, 2014. 32 p.

24. Chava S. V. Investigation of the peripheral organs of the immune system when introducing a new generation of immunomodulators into the body. Morphology. 2006. No. 4 P. 133.

25. Churina E. G. Factors of immunosuppression in various pathologies Bulletin of Siberian medicine.2011 No. 4-pp. 103-111.

26. Sharshembiev D. A. M. A. Sabirov. Morphology of the thymus under conditions of immunostimulation. Bulletin of the KRSU. 2007. No. 9. pp. 3-5.

27. Yarilin A. A. Cytokines in the thymus.Biological activity and functions of cytokines in the thymus. A. A. Yarilin Cytokines and inflammation. 2003. No. 2. pp. 3-11.

28. Yarilin A. A. T-cells are recent emigrants from the thymus. Immunology. - 2012. Vol. 33, No. 6. - p. 326-334.

29. Yarilin A. A. Neuroimmunoendocrinology of the Handbook of neuroimmunoendocrinology. - Moscow: Meditsina, 2008. - pp. 299-345.

30. Yaroshenko I. F., Pisarev V. B. Fundamentals of immunopathology. Volgograd.2001-p. 168.

31. Dominguez-Herpe L. Changes induced by chronic stress in blood lymphocytic subsets and primary and secondary immune organs of mice / L. DominguezHerpe, M. Rey-Mendes BMC Immunol. 2001. Vol. 2, No. 1. P. 7.

32. Goncharov N. P. Dehydroepiandrosterone: properties, metabolism, biological significance $M$ : Adamant, 2004. - 158c.

33. Hsieh With S. H.-M. Lee, C. W. J. Lio. Selection of regulatory $T$ cells in the thymus Nature reviews. Immunology.2012. Volume 12. P. 157-167.

34. Huseynov T. S. et al., 2005; E. H. Sibilaeva, 2008; A. N. Nurmukhambetov et al., 2011).

35. Ismail Naser Nawal Hasan Indicators of the level of serum thymic activity in the umbilical cord blood of newborns: Dis. . Cand. med nauk. - M., 2003. - 16 p. 
36. Khasanova D. A., Teshaevsh. Ya. Topographical and anatomical features of the lymphoid structures of the small intestine of rats in normal and against the background of chronic radiation diseases/ / European science review Vienna, Austria No. 9-10 2018, Volume 2. Medical science P. 197-198.

37. Khaydarov F. G., Khasanova D. A. The study of behavioral and morphological disorders in animals with modeled pathology of mild traumatic brain injury/ / American Journal of Medicine and Medical Sciences.- 2020.-№10 (10) 9, 803-807.

38. Mak-Jillc. Et al. Fetal exposure to ethanol has long-term effects on the severity of influenza virus infections. McGill, K. D. Meyerholz, M. Edsen-Moore et al. //J. Immunol. 2009.Volume.182. No. 12. p. 7803-7808.

39. Shteynman G. Changes in the human thymus in agig / / Human thymus. Edited by $\mathrm{H}$. Mullre-Hermelink. Berlin, 1986, pp. 43-89.

40. Sun D. P. [et al.] Thymus hyperplasia after chemotherapy in adults with mature B-cell lymphoma and its effect on thymus output and CD4 (+) T cell repopulation. Oncoimmunology. 2016. Volume 18 No. 5( 5). p. 1137417. 